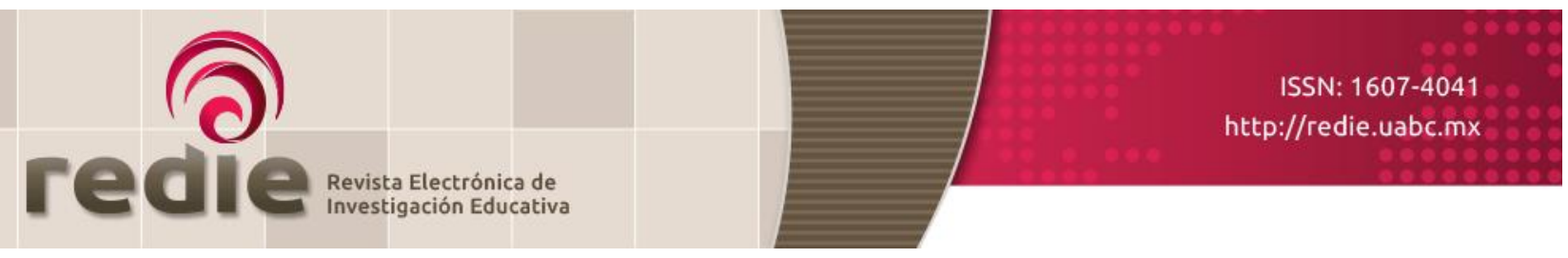

Vol. 19, Núm. 3, 2017

\title{
Estudio del tratamiento del enlace químico en los libros de texto españoles
}

\section{Approach to Chemical Bonding in Spanish Textbooks}

\author{
María Esther González-Felipe (1) mesther2202gf@hotmail.com \\ Constancio Aguirre-Pérez (2) constancio.aguirres@uclm.es \\ José Manuel Cortes-Simarro (2) josemanuel.cortes@uclm.es \\ Raquel Fernández Cezar (2) raquel.fcezar@uclm.es \\ Ana María Vázquez Monilí (2) ana.vazquez@uclm.es \\ (1) I.E.S. Bachiller Sabuco de Albacete \\ (2) Universidad de Castilla-La Mancha \\ (Recibido: 21 de mayo de 2015; Aceptado para su publicación: 18 de diciembre de 2015)
}

Cómo citar: González-Felipe, M. E., Aguirre-Pérez, C., Cortes-Simarro, J. M., Fernández, R. y Vázquez, A. M. (2017). Estudio del tratamiento del enlace químico en los libros de texto españoles. Revista Electrónica de Investigación Educativa, 19(3), 60-72. https://doi.org/10.24320/redie.2017.19.3.1184

\section{Resumen}

En este trabajo se presenta un análisis del tratamiento del concepto de enlace químico en los libros de texto de Educación Secundaria en España. Se ha realizado un estudio de caso comparativo de tres de las editoriales más utilizadas con mayor frecuencia, para ello se elaboró una rúbrica con seis categorías: secuenciación de los contenidos, causa de la formación del enlace, terminología utilizada, clasificación de las sustancias según tipo de enlace, modelos de enlace y estudio de las fuerzas intermoleculares. Se concluye que entre las editoriales no hay diferencias en cuatro de los seis puntos, pero sí en: terminología empleada y el tratamiento de las fuerzas intermoleculares. Se aconseja al profesorado hacer uso de la rúbrica diseñada para analizar el tratamiento del enlace químico en los libros de texto y decidir cuál utilizar en sus clases.

Palabras clave: Libros de texto, enlace químico, concepciones alternativas, dificultades de aprendizaje.

\section{Abstract}

This work presents an analysis of the approach taken by secondary education textbooks in Spain to the concept of chemical bonding. A comparative case study was performed on three of the most widely used publishers, for which a rubric was devised with six categories: content sequencing, cause of bond formation, terminology used, substance classification based on type of bond, chemical bonding models and the study of intermolecular forces. The conclusion is that there are no differences between publishers in four of the six aspects, but differences do exist in the terminology used and the approach to intermolecular forces. Teachers are advised to make use of the rubric to analyze textbooks' approach to chemical bonding and decide which to use in their classes. 
Keywords:Textbooks, chemical bond, misconceptions, learning difficulties.

\section{Introducción}

El concepto de enlace químico es uno de los más abstractos y complejos de la Química (Levy Nahum, Mamlok-Noaman, Hofstein y Kronik, 2010) siendo, al mismo tiempo, un concepto clave y fundamental, ya que permite responder a diversas cuestiones como:

- La fórmula química y estequiometría determinada de cada sustancia o compuesto químico.

- La justificación de las propiedades físicas y químicas de las distintas sustancias.

- La existencia de átomos en estado libre, moléculas o estructuras cristalinas.

- Las clases de uniones que se rompen y se forman en el transcurso de las reacciones químicas.

El enlace químico podría ser considerado un "concepto estructurante", según la terminología de Gagliardi y Giordan (1986); sin embargo, es considerado por alumnos y profesores un concepto "muy complicado" (Taber, 2001; Riboldi, Pliego y Odetti, 2004). La comprensión del enlace es básica para que los alumnos comprendan y expliquen las propiedades físicas y químicas de las sustancias y la forma en la que se unen las partículas, así como la importancia que tiene esto en la síntesis de nuevos compuestos y su incidencia en la mejora de la calidad de vida.

Los libros de texto son los recursos materiales más ampliamente utilizados por los profesores en la enseñanza secundaria, y en numerosas ocasiones el único (Calvo y Martín, 2005). Los libros de texto influyen en lo que los profesores enseñan y, por lo tanto, en cómo aprenden los estudiantes (Fernández, Jiménez y Solano, 1997), por lo que el análisis de los libros de texto es de suma importancia (Justi y Gilbert, 2003).

La forma en que la química se presenta en los libros de texto de todo el mundo ha sido estudiada por diversos autores en las últimas décadas (Abraham, Grzybowski, Renner y Marek, 1992; Matus, Benarroch y Nappa, 2011; Matus, Benarroch y Perales, 2008). Calvo y Martín (2005), quienes analizaron el tratamiento dado a la Química en los libros de texto de Educación Secundaria Obligatoria (ESO) con el objetivo de determinar si se adaptaban adecuadamente al currículo oficial, señalan que no hay gran diferencia en la secuenciación de los contenidos entre editoriales y destacan la frecuencia con que aparecen imprecisiones o inexactitudes o incluso errores químicos, como no incluir los últimos elementos del Sistema Periódico (SP), utilizar la nomenclatura antigua para nombrar los grupos del SP o no diferenciar claramente entre sustancia pura y mezcla, o entre los distintos tipos de mezclas. Furió y Guisasola (1997) estudiaron las visiones distorsionadas de la ciencia transmitida por los libros de texto, así como las carencias epistemológicas de los mismos.

A pesar de la importancia del concepto de enlace químico en la enseñanza de la Química, el análisis del tratamiento dado al mismo en los libros de texto no ha recibido mucha atención, particularmente en el mundo de habla hispana. Existen estudios relacionados con el tratamiento del enlace químico en los libros de texto (Ashkenazi y Kosloff, 2006; Hurst, 2002; Justi y Gilbert, 2003). Solbes y Vilches (1991) encontraron que los libros de texto suelen presentar los modelos de enlace químico como descripciones reales más que como representaciones mentales. Además, se ha comprobado que los modelos de enlace químico que se presentan en los libros de texto influyen en las concepciones alternativas que presentan los estudiantes (Bergqvist, Drechsler, De Jong y Rundgren, 2013). De Posada (1999) se centró en el tratamiento del enlace metálico en los libros de texto indicando que pocos libros de texto aportan pruebas sobre la realidad de los iones y sus diferencias con los átomos. Matus et al. $(2008 ; 2011)$ centraron su investigación en las imágenes usadas para la enseñanza del enlace químico en libros de texto argentinos y concluyeron que el grado de iconicidad o referencialidad disminuye a medida que aumenta la edad de los destinatarios, al tiempo que el lenguaje gráfico es sustituido paulatinamente por lenguajes más formales y el contenido conceptual de la imagen se va enriqueciendo.

Levy et al. (2008) elaboraron un esquema ilustrativo del enfoque tradicional que se suele dar en los libros de texto al enlace químico. En la mayoría de ellos, los diferentes tipos de enlace se presentan como 
entidades independientes. Este enfoque se explica por motivos históricos, pero se realiza a costa de establecer simplificaciones y generalizaciones que terminan por convertirse en "impedimentos de aprendizaje". En la tabla I se presentan algunos impedimentos pedagógicos, señalados por Levy et al. (2008), relacionados con la enseñanza tradicional del concepto del enlace químico.

Tabla I. Impedimentos pedagógicos atribuibles a la enseñanza tradicional del enlace químico

\begin{tabular}{|c|c|}
\hline $\begin{array}{l}\text { Enlace covalente } \\
\text { vs } \\
\text { enlace iónico }\end{array}$ & $\begin{array}{l}\text { - Se presentan de forma dicotómica: Compartición/transferencia de electrones. } \\
\text { - Se excluye la realidad de una escala continua covalente-iónica. } \\
\text { - Aunque los enlaces covalente puros existen entre átomos idénticos no sucede lo mismo } \\
\text { con los iónicos, ya que todos se encuadran dentro de una escala de diferentes } \\
\text { electronegatividades (EN). }\end{array}$ \\
\hline $\begin{array}{l}\text { Electronegatividad } \\
\text { y polaridad } \\
\text { del enlace }\end{array}$ & $\begin{array}{l}\text { - Se explica el concepto sólo en el contexto de la predicción del carácter polar o apolar del } \\
\text { enlace o de su carácter iónico o covalente. } \\
\text { - Se obvia la existencia comprobada experimentalmente de enlaces de marcado carácter } \\
\text { covalente entre átomos con grandes diferencias en sus EN. }\end{array}$ \\
\hline La "regla" del octeto & $\begin{array}{l}\text { - Relegando su naturaleza meramente algorítmica o instrumental, se presenta como } \\
\text { condición obligatoria para formar enlaces propiamente dichos y, además, con carácter } \\
\text { "explicativo" de los mismos. }\end{array}$ \\
\hline Enlace metálico & $\begin{array}{l}\text { - Explicación en función de sus propiedades físicas y químicas obviando que esas } \\
\text { propiedades no son comunes a todos los metales, existiendo una gran variabilidad en esos } \\
\text { parámetros (Tf, Tb, brillo, conductividad térmica o eléctrica, maleabilidad, ductilidad, etc.). } \\
\text { - Se suele hacer referencia al modelo de "iones flotando en un mar de electrones" que se } \\
\text { presenta como un modelo completamente diferente al del enlace covalente por } \\
\text { "compartición" de electrones, cuando la realidad también se puede explicar en términos } \\
\text { de una escala continua que incluye diferentes grados de deslocalización electrónica. }\end{array}$ \\
\hline $\begin{array}{l}\text { Fuerzas } \\
\text { intermoleculares }\end{array}$ & $\begin{array}{l}\text { - Se les presenta como simples "fuerzas" en contraposición al término de enlace sin tener } \\
\text { en cuenta la fuerza relativa de los diferentes tipos de enlace y su importancia, ya que } \\
\text { incluso las débiles "fuerzas" de unión pueden tener importantes consecuencias químicas } \\
\text { (bioquímica). } \\
\text { - Se reduce el enlace de H tan sólo cuando se da entre el H y los átomos de de N, O y F } \\
\text { cuando se pueden dar entre otros átomos o grupos de átomos. }\end{array}$ \\
\hline
\end{tabular}

Como se ha indicado hasta ahora, los libros de texto pueden ser una importante fuente de adquisición de errores conceptuales. Muchos de estos errores resultan del uso en los libros de texto de modelos "sobresimplificados" y de metodologías tradicionales que presentan una imagen limitada e incorrecta del enlace químico (Levy, Mamlok-Noaman, Hofstein y Taber 2010). En este sentido, un análisis del tratamiento que se da a la información en los libros de texto puede ayudar a entender los errores conceptuales de los alumnos. Teniendo en cuenta el papel crucial de los libros de texto en la enseñanza de la química, y más concretamente del enlace químico, se llevó a cabo el presente trabajo.

\section{Metodología}

Se aborda la investigación mediante un estudio de caso de tipo comparativo y analítico en el que cada caso lo constituye el análisis del tratamiento del enlace químico que hace un determinado libro de texto de una editorial y de un curso o nivel concreto. En el currículum español, el enlace químico se trata en los distintos niveles educativos desde 30. de ESO hasta 20. de Bachillerato, pero con distinto grado de profundidad siguiendo un modelo en espiral (De Posada, 1999). Por ello, el estudio realizado incluye todos estos niveles educativos. Los libros de texto -elegidos entre los más ampliamente utilizados en España- se enlistan en la tabla II. 
Tabla II. Libros de texto analizados y códigos asignados a cada uno de ellos

\begin{tabular}{lcclcc}
\hline Editorial & Nivel & Año & \multicolumn{1}{c}{ Autor } & ISBN & Código \\
\hline Santillana & 3o. ESO & 2010 & Rosa Marín & $978-84-294-3027-1$ & 1 \\
Oxford & 3o. ESO & 2010 & Isabel Piñar & $978-84-673-5334-0$ & 2 \\
Santillana & 40. ESO & 2008 & Rosa Marín & $978-84-294-0984-0$ & 3 \\
Oxford & 40. ESO & 2008 & Isabel Piñar & $978-84-673-3859-1$ & 4 \\
Edebé & 40. ESO & 2008 & Antonio Garrido & $978-84-236-8753-4$ & 5 \\
Santillana & 10. BAC & 2008 & Rosa Marín & $978-84-294-0987-1$ & 6 \\
Oxford & 10. BAC & 2008 & Mario Ballesteros y Jorge Barrio & $978-84-673-3881-2$ & 7 \\
Edebé & 10. BAC & 2008 & Antonio Garrido & $978-84-236-8590-5$ & 8 \\
Santillana & 20. BAC & 2009 & Rosa Marín & $978-84-294-0993-2$ & 9 \\
Oxford & 20. BAC & 2009 & Jaime Peña y Ma. Carmen Vidal & $978-84-673-5098-2$ & 10 \\
Edebé & 20. BAC & 2009 & Antonio Garrido & $978-84-236-9282-8$ & 11 \\
\hline
\end{tabular}

Рara realizar el estudio se asignó un código numérico a cada libro de texto, se seleccionaron los temas que hacían referencia al enlace químico y se sometieron a un primer análisis cualitativo de forma individualizada. Se elaboró una rúbrica de análisis compuesta por seis categorías determinadas de acuerdo con los primeros datos obtenidos y la bibliografía referente a las dificultades de aprendizaje de los estudiantes. Las seis categorías consideradas fueron: secuenciación de los contenidos, causas de la formación de enlaces, terminología utilizada, clasificación de las sustancias según tipo de enlace, modelos de enlace utilizados y estudio de las fuerzas intermoleculares. Posteriormente, con el objetivo de realizar un análisis más profundo se elaboró un cuestionario con ítems correspondientes a cada una de las categorías incluidas en la rúbrica. El cuestionario correspondiente a cada una de estas categorías se muestra en las tablas III a VIII. Dos investigadores completaron el cuestionario individualmente. El resultado del análisis realizado por estos investigadores fue coincidente al $90 \%$. Posteriormente las diferencias fueron analizadas por los autores y se llegó a un acuerdo sobre las mismas.

\section{Resultados y discusión}

A continuación se discuten los resultados obtenidos tras usar la rúbrica elaborada en el análisis de los temas de enlace químico incluidos en los libros de texto analizados.

\subsection{Secuenciación de los contenidos}

Tabla III. Análisis de los libros de texto en relación a la secuenciación de contenidos

\begin{tabular}{|c|c|c|c|c|c|c|c|c|c|c|c|}
\hline \multirow{2}{*}{ ítems } & \multicolumn{11}{|c|}{ Códigos } \\
\hline & 1 & 2 & 3 & 4 & 5 & 6 & 7 & 8 & 9 & 10 & 11 \\
\hline 1 Dedica un tema al estudio del enlace químico & & & & $\mathrm{X}$ & & $\mathrm{X}$ & $\mathrm{X}$ & $\mathrm{X}$ & $\mathrm{X}$ & $\mathrm{X}$ & $\mathrm{X}$ \\
\hline 2 El enlace químico forma parte de un tema más amplio & $\mathrm{x}$ & $\mathrm{x}$ & $\mathrm{x}$ & & $\mathrm{x}$ & & & & & & \\
\hline $\begin{array}{l}3 \text { Explica las propiedades de las sustancias y a continuación los } \\
\text { diferentes enlaces que presentan }\end{array}$ & & & & & & & & & & & \\
\hline $\begin{array}{l}4 \text { Explica las propiedades a continuación de explicar cada tipo } \\
\text { de enlace }\end{array}$ & & & & $\mathrm{x}$ & $\mathrm{x}$ & $\mathrm{x}$ & $\mathrm{x}$ & & $\mathrm{x}$ & $\mathrm{x}$ & \\
\hline $\begin{array}{l}5 \text { Explica los tipos de enlace y a continuación presenta las } \\
\text { propiedades de las sustancias con cada tipo de enlace }\end{array}$ & & & $\mathrm{X}$ & & & & & $\mathrm{X}$ & & & $\mathrm{X}$ \\
\hline 6 Inicia el estudio de los tipos de enlace con el enlace iónico & & & $x$ & & $\mathrm{x}$ & $\mathrm{x}$ & $x$ & $\mathrm{x}$ & $\mathrm{x}$ & $x$ & $\mathrm{x}$ \\
\hline 7 Inicia el estudio de los tipos de enlace con el enlace covalente & & & & $x$ & & & & & & & \\
\hline 8 Inicia el estudio de los tipos de enlace con el enlace metálico & & & & & & & & & & & \\
\hline
\end{tabular}

Al igual que señalan Calvo y Martín (2005) en su estudio sobre el tratamiento de la Química en los libros de texto, no hay gran diferencia en la secuenciación entre editoriales. Ninguna de las editoriales dedica un tema al estudio del enlace químico en 3o. ESO, tratándolo como un apartado dentro de otro tema, y solamente la editorial Oxford le dedica un tema en 40. ESO. En 10. y 20. de Bachillerato todas las editoriales dedican un tema al estudio del enlace químico. Los libros de 4o. de ESo (código 3), y 1o. y 20. de Bachillerato (códigos 8 y 11) presentan los distintos tipos de enlace y a continuación estudian las propiedades de las sustancias; el resto presenta las propiedades de las sustancias después de cada tipo de 
enlace, pero ninguno de los libros comienza con el estudio de las propiedades de las sustancias. Es de destacar que la única editorial que utiliza la misma secuenciación en todos los cursos es Oxford.

Todos los libros comienzan el estudio del enlace con el enlace iónico, excepto uno (código 4) que lo hace con el enlace covalente. Ninguno de los libros analizados comienza con el estudio del enlace metálico. Esto no está de acuerdo con lo señalado por Bergvist, Drechsler, De Jong y Rundgren (2013), quienes indican que comenzar el estudio del enlace con el enlace covalente es una práctica común. Según Taber (2001), se debería comenzar con el estudio del enlace metálico, cosa que no hace ninguno de los libros analizados, continuar con el iónico y finalizar con el covalente, para estudiar de esta forma en primer lugar los cristales (metálico e iónico) y terminar con las moléculas discretas covalentes, para evitar el error conceptual frecuentemente presentado por los estudiantes de pensar en la existencia real de moléculas iónicas y de que los cristales iónicos están formados por moléculas.

\subsection{Causa de la formación del enlace}

Tabla IV. Análisis de los libros de textos en relación con la causa de la formación del enlace

\begin{tabular}{|c|c|c|c|c|c|c|c|c|c|c|c|}
\hline \multirow{2}{*}{ Ítems } & \multicolumn{11}{|c|}{ Códigos } \\
\hline & 1 & 2 & 3 & 4 & 5 & 6 & 7 & 8 & 9 & 10 & 11 \\
\hline $\begin{array}{l}1 \text { Relaciona la estabilidad de las sustancias con la formación del } \\
\text { enlace }\end{array}$ & & & & & & & $X$ & $X$ & X & $x$ & $X$ \\
\hline 2 Relaciona la estabilidad de las sustancias con la regla del octeto & & $x$ & & & & & $x$ & & $x$ & & $x$ \\
\hline 3 Los átomos se enlazan para cumplir la regla del octeto & & & & & & $x$ & $x$ & $x$ & & & $x$ \\
\hline 4 Los átomos se enlazan para adquirir configuraciones estables & & & & $x$ & & & $\mathrm{X}$ & & & & $x$ \\
\hline $\begin{array}{l}5 \text { Los átomos se enlazan para adquirir un estado de mínima } \\
\text { energía }\end{array}$ & & & & & $X$ & & & $x$ & $\mathrm{X}$ & $x$ & $X$ \\
\hline $\begin{array}{l}6 \text { Relaciona estabilidad de las sustancia con un estado de mínima } \\
\text { energía }\end{array}$ & & & & & & & & $X$ & & $x$ & $X$ \\
\hline 7 Relaciona distancia de enlace con un estado de mínima energía & & & & $x$ & & $x$ & $x$ & & $x$ & $x$ & $x$ \\
\hline
\end{tabular}

Generalmente no se presentan las causas de la formación de enlaces y se justifica la formación del enlace solamente por la tendencia de los átomos a adquirir la configuración electrónica de gas noble o cumplir la regla del octeto. Únicamente 4 de los libros analizados, tres de Bachillerato (códigos 8, 9 y 10) y uno de 40. de ESO (código 5), señalan que la causa de la formación del enlace es la "minimización de la energía", y sólo 3 (códigos 8, 10 y 11) relacionan la estabilidad de las sustancias con el estado de mínima energía. Por ejemplo, en el libro de código 8 se dice: "Los átomos se unen para formar agrupaciones de mayor estabilidad y menor energía que la que tenían los átomos por separado" y en el libro de código 11 se dice: "Рara que se forme cualquier tipo de enlace estable, el sistema resultante debe tener menor energía que el que constituían las partículas aisladas. Es más, cuanto mayor sea la disminución de energía mayor será la estabilidad del enlace y del sistema formado".

De acuerdo con Levy et al. (2010) la interrogante principal debería ser: ¿Cuál es la causa de que los átomos interaccionen y formen un enlace? No plantear esta interrogante lleva a los alumnos a pensar que la formación del enlace es algo totalmente "misterioso" e "inexplicable", cuando no hay nada de misterioso en la formación del enlace químico si se introduce en primer lugar la naturaleza eléctrica de las partículas químicas (átomos, iones y moléculas) y se explica que el enlace es la consecuencia de las atracciones y repulsiones entre núcleos y electrones y se introduce el concepto de estabilidad que corresponde al estado de mínima energía que se da cuando las cargas opuestas están lo más cerca posible y las iguales lo más lejos.

Prácticamente todos los libros explican la formación del enlace iónico en términos de transferencia de electrones y la del enlace covalente en términos de compartición de electrones. Ninguno de los libros analizados emplea la existencia de fuerzas electrostáticas para explicar la formación de todos los tipos de enlace. La mayoría habla de fuerzas electrostáticas en el caso del enlace iónico pero nunca en el caso de los enlaces covalente y metálico. Esta forma de presentar tanto el enlace iónico como el covalente ha sido fuertemente criticada por miembros de la comunidad científica (Bergvist, Drechsler, De Jong y Rundgren, 
2013) al considerar que explicar el enlace iónico como un proceso de transferencia de electrones y no como un equilibrio de fuerzas puede llevar a los alumnos a interpretar que el enlace iónico solamente existe entre átomos que han transferido electrones entre ellos (Taber, 2001), y que las fuerzas de atracción en un cristal iónico sólo se dan entre pares de iones y no entre cada ion y todos sus vecinos en la red.

\subsection{Terminología utilizada}

Tabla V. Análisis de los libros de textos en relación a la terminología utilizada

\begin{tabular}{|c|c|c|c|c|c|c|c|c|c|c|c|c|}
\hline \multirow{2}{*}{\multicolumn{2}{|c|}{ Ítems }} & \multicolumn{11}{|c|}{ Códigos } \\
\hline & & 1 & 2 & 3 & 4 & 5 & 6 & 7 & 8 & 9 & 10 & 11 \\
\hline 1 & Define las moléculas como agrupaciones de átomos & $\mathrm{X}$ & $x$ & & & $\mathrm{X}$ & $\mathrm{X}$ & & & & & \\
\hline 2 & Define sustancia molecular como conjunto de moléculas & $X$ & & & & & & & & & & \\
\hline 3 & $\begin{array}{l}\text { Define molécula como conjunto de átomos unidos por } \\
\text { enlace covalente }\end{array}$ & & & $X$ & $X$ & & & & $x$ & & & \\
\hline 4 & Presenta los cristales como un tipo de sustancias & $x$ & $x$ & $x$ & & & $x$ & & & & & \\
\hline 5 & $\begin{array}{l}\text { Utiliza distintas terminología para referirse a un mismo } \\
\text { concepto (cristal, estructura cristalina, sólido cristalino, } \\
\text { redes cristalinas) }\end{array}$ & & $x$ & $X$ & $\mathrm{X}$ & X & $X$ & $x$ & $x$ & $x$ & $X$ & $x$ \\
\hline 6 & $\begin{array}{l}\text { Define enlace como unión entre átomos sin especificar su } \\
\text { naturaleza }\end{array}$ & & & & & & $X$ & & & & & \\
\hline 7 & $\begin{array}{l}\text { Define enlace como unión entre átomos por fuerzas } \\
\text { electrostáticas }\end{array}$ & & & $X$ & $x$ & & & $x$ & $X$ & & & \\
\hline 8 & $\begin{array}{l}\text { Define enlace como fuerzas que mantienen unidas a los } \\
\text { átomos y a las moléculas }\end{array}$ & & & & & $X$ & & & & & $x$ & \\
\hline 9 & Define enlace covalente como la unión entre átomos & & & & $X$ & $X$ & & & $x$ & $x$ & & $x$ \\
\hline 10 & Define enlace metálico como la unión entre átomos & & & & & & & & $x$ & & & \\
\hline 11 & Define enlace iónico como la unión entre iones & & & $x$ & $x$ & X & & $x$ & $x$ & $x$ & $x$ & $x$ \\
\hline 12 & $\begin{array}{l}\text { Define fuerzas intermoleculares como la unión entre } \\
\text { moléculas }\end{array}$ & & & & & & & $x$ & $X$ & $x$ & $x$ & \\
\hline 13 & Define enlace como fuerza de atracción entre átomos & & & $x$ & $x$ & & & $x$ & & & & \\
\hline 14 & Se diferencia enlace covalente polar y covalente apolar & & & & & & $x$ & $x$ & $x$ & $x$ & $x$ & $x$ \\
\hline 15 & $\begin{array}{l}\text { Introduce el concepto de molécula dipolo pero no define } \\
\text { momento dipolar }\end{array}$ & & & & & & $x$ & & & & & \\
\hline 16 & Define dipolo eléctrico o momento dipolar & & $x$ & & & & & $x$ & $x$ & $X$ & $X$ & $x$ \\
\hline 17 & $\begin{array}{l}\text { Define enlace iónico puro como un caso límite de enlace } \\
\text { covalente polar }\end{array}$ & & & & & & & $x$ & & & & \\
\hline 18 & $\begin{array}{l}\text { No define enlace químico, aunque habla de la combinación } \\
\text { de átomos del mismo o diferentes elementos }\end{array}$ & $x$ & $x$ & & & & & & & & & \\
\hline 19 & $\begin{array}{l}\text { Define enlace como fuerzas que mantienen unidas a los } \\
\text { átomos, iones o moléculas }\end{array}$ & & & & & & & & $X$ & $x$ & & $x$ \\
\hline
\end{tabular}

Se observa gran confusión en la terminología utilizada. Todos los libros excepto uno (código 1) utilizan distinta terminología para referirse a un mismo concepto. Asimismo, se observa que una misma editorial utiliza diferentes definiciones y términos para un mismo concepto en las diferentes etapas educativas.

Es frecuente el uso de términos que no se definen y no se aclaran, o que se definen muy posteriormente. Por ejemplo, en el libro con código 3 se dice: "Los compuestos iónicos son sólidos cristalinos"; pero no define sólido cristalino. Más adelante introduce el concepto de cristal y dentro del mismo incluye los cristales iónicos diciendo: "Cristales iónicos: las partículas que forman el cristal son iones". En este caso se utilizan indistintamente los términos sólido cristalino y cristal, pero sin indicarlo previamente. ¿Se da por entendido que cristal y sólido cristalino son sinónimos? ¿No quedaría mucho más claro si se dijera "los compuestos iónicos son sólidos cristalinos o cristales"?

La terminología confusa puede inducir a errores conceptuales en los alumnos, por ejemplo, llamar "sustancias formadas por átomos" a los cristales atómicos covalentes frente a las "sustancias formadas por moléculas", puede llevar a pensar que estas últimas no están formadas, en último término, también por átomos. 
Cada editorial utiliza distinta terminología para designar el mismo concepto; así, por ejemplo, la editorial Santillana utiliza el término "sustancias simples" para designar a las sustancias formadas por moléculas de elementos como el $\mathrm{O}_{2}, \mathrm{~N}_{2}$, etc., mientras que la editorial Oxford emplea el término "elementos moleculares". Las uniones entre moléculas se designan como fuerzas intermoleculares, uniones intermoleculares o enlaces intermoleculares dependiendo de la editorial. En otras ocasiones se utiliza una terminología complicada en lugar de intentar simplificar al máximo los términos utilizados, por ejemplo "compuestos covalentes reticulares" o "cristales moleculares covalentes", empleados en el libro con código 4. Esto puede crear confusión en los alumnos, sobre todo si se utiliza en los niveles de educación inferiores. Complica más la situación el hecho de que frecuentemente se utiliza distinta terminología en casos en los que podría utilizarse la misma: por ejemplo, en el caso de un cristal iónico se habla de red cristalina iónica, mientras que para el metálico se habla de cristal metálico (libro con código 4). Los cristales atómicos covalentes, por ejemplo el diamante, se nombran como "sólidos covalentes cristalinos" (código 7), "cristales atómicos covalentes" (código 4) o incluso "sólidos reticulares" (código 11). Homologar la terminología utilizada ayudará a evitar confusiones en los alumnos.

\subsection{Clasificación de las sustancias según tipo de enlace}

Tabla VI. Análisis de los libros de textos en relación a la clasificación de las sustancias y los tipos de enlace

\begin{tabular}{|c|c|c|c|c|c|c|c|c|c|c|c|c|}
\hline \multirow{2}{*}{\multicolumn{2}{|c|}{ ítems }} & \multicolumn{11}{|c|}{ Códigos } \\
\hline & & 1 & 2 & 3 & 4 & 5 & 6 & 7 & 8 & 9 & 10 & 11 \\
\hline 1 & Clasifica los cristales en iónicos, covalentes y metálicos & $\mathrm{X}$ & & $\mathrm{X}$ & & & & & & & & \\
\hline 2 & $\begin{array}{l}\text { Clasifica los cristales en iónicos, covalente, metálicos } \\
\text { y moleculares }\end{array}$ & & & & & & & & & & & \\
\hline 3 & $\begin{array}{l}\text { Clasifica las sustancias como átomos aislados, sustancias } \\
\text { moleculares y cristales }\end{array}$ & $x$ & & $X$ & & & & & & & & \\
\hline 4 & $\begin{array}{l}\text { Clasifica las sustancias como agrupación de los átomos } \\
\text { en los elementos y agrupación de los átomos en los } \\
\text { compuestos }\end{array}$ & & $X$ & & & & & & & & & \\
\hline 5 & $\begin{array}{l}\text { Clasifica los elementos como átomos aislados, moléculas } \\
\text { y cristales }\end{array}$ & & $X$ & & & & & & & & & \\
\hline 6 & Clasifica los compuestos en moléculas y cristales & & $x$ & & & & & & & & & \\
\hline 7 & $\begin{array}{l}\text { Clasifica las sustancias covalentes en moléculas y cristales } \\
\text { atómicos covalentes }\end{array}$ & & & & & $X$ & & & & $X$ & $\mathrm{x}$ & \\
\hline 8 & $\begin{array}{l}\text { Clasifica los cristales en iónicos, covalentes moleculares, } \\
\text { atómicos covalentes y metálicos }\end{array}$ & & & & $X$ & & $X$ & & $x$ & & & $x$ \\
\hline 9 & $\begin{array}{l}\text { Clasifica los enlace en tres tipos: iónico, covalente } \\
\text { y metálico }\end{array}$ & & & $X$ & $X$ & $x$ & $x$ & $x$ & & & $X$ & \\
\hline 10 & $\begin{array}{l}\text { Clasifica los enlaces en iónico, covalente, metálico } \\
\text { y fuerzas intermoleculares }\end{array}$ & & & & & & & & $x$ & $X$ & & $X$ \\
\hline
\end{tabular}

Una misma editorial utiliza distintas definiciones y criterios de clasificación de los enlaces, dependiendo del curso en el que trate el tema. Los distintos criterios de clasificación utilizados, según los cursos y editoriales, dan lugar a clasificaciones muy diferentes y dispares: desde la situación más simple, elementos y compuestos, hasta la más compleja que incluye siete tipos de sustancias: moléculas en las sustancias simples, cristales atómicos covalentes, cristales covalentes moleculares, compuestos covalentes moleculares, compuestos covalentes reticulares, compuestos iónicos y metales. Esta gran disparidad de criterios puede llevar a pensar a los alumnos a que lo estudiado en los cursos anteriores deja de ser válido al pasar a un curso superior.

La mayoría de los libros clasifica los enlaces en tres tipos: iónico, covalente y metálico, y sólo tres (códigos 8,9 y 11) incluyen las fuerzas intermoleculares como un tipo de enlace.

Para evitar esta confusión las sustancias pueden ser clasificadas en reticulares y no reticulares. Dentro de las sustancias reticulares hay tres tipos: las metálicas, las iónicas y las covalentes. Las sustancias no reticulares pueden ser moleculares y atómicas. Sólo los gases nobles se presentan como átomos sueltos. 


\subsection{Modelos de enlace}

Tabla VII. Análisis de los libros de textos en relación a los modelos de enlace utilizados

\begin{tabular}{|c|c|c|c|c|c|c|c|c|c|c|c|c|}
\hline \multirow{2}{*}{\multicolumn{2}{|c|}{ Ítems }} & \multicolumn{11}{|c|}{ Códigos } \\
\hline & & 1 & 2 & 3 & 4 & 5 & 6 & 7 & 8 & 9 & 10 & 11 \\
\hline 1 & $\begin{array}{l}\text { Explica el enlace iónico mediante transferencia de } \\
\text { electrones }\end{array}$ & & & $x$ & $x$ & $x$ & $x$ & $X$ & $X$ & $x$ & & $X$ \\
\hline 2 & $\begin{array}{l}\text { Explica el enlace covalente por compartición de } \\
\text { electrones }\end{array}$ & & & $x$ & $x$ & $x$ & $x$ & $X$ & $x$ & $x$ & $X$ & $x$ \\
\hline 3 & $\begin{array}{l}\text { Ejemplo usado para el estudio del enlace iónico: cloruro } \\
\text { de sodio }\end{array}$ & & & $X$ & $X$ & & $x$ & & $x$ & $x$ & $X$ & \\
\hline 4 & $\begin{array}{l}\text { Ejemplo usado para el estudio del enlace iónico distinto } \\
\text { al } \mathrm{NaCl}\end{array}$ & & & $x$ & & $x$ & & $X$ & & & & $x$ \\
\hline 5 & Explica el ciclo de Born-Haber & & & & & & & $X$ & $x$ & $x$ & $X$ & $x$ \\
\hline 6 & Utiliza el diagrama de Lewis para el enlace covalente & & & $x$ & $x$ & $x$ & & $x$ & $x$ & $x$ & & $x$ \\
\hline 7 & $\begin{array}{l}\text { Utiliza el diagrama de Lewis para el enlace covalente } \\
\text { y el iónico }\end{array}$ & & & & & & & & & & $X$ & \\
\hline 8 & Utiliza la TEV para el estudio del enlace covalente & & & & & & & & $x$ & & 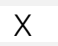 & $x$ \\
\hline 9 & Utiliza la TRPECV para el estudio del enlace covalente & & & & & & & & & $x$ & $x$ & $x$ \\
\hline 10 & $\begin{array}{l}\text { Utiliza la teoría de hibridación en el estudio del enlace } \\
\text { covalente }\end{array}$ & & & & & & & & & $X$ & $X$ & $x$ \\
\hline 11 & $\begin{array}{l}\text { Utiliza la teoría de orbitales moleculares para el estudio } \\
\text { del enlace covalente }\end{array}$ & & & & & & & & & & & \\
\hline 12 & $\begin{array}{l}\text { Explica el enlace metálico como atracción entre } \\
\text { electrones de valencia y los iones positivos }\end{array}$ & & & $x$ & $x$ & $x$ & & & $X$ & $X$ & & \\
\hline 13 & $\begin{array}{l}\text { Explica el enlace metálico como pérdida de electrones } \\
\text { para adquirir configuración de gas noble }\end{array}$ & & & & & & 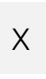 & & & $X$ & & \\
\hline 14 & $\begin{array}{l}\text { Explica el enlace metálico mediante el mar de } \\
\text { electrones de Drude }\end{array}$ & & & & & & & $X$ & & & $X$ & $X$ \\
\hline 15 & Explica el enlace metálico mediante la teoría de bandas & & & & & & & & & $x$ & $X$ & $X$ \\
\hline
\end{tabular}

Sin duda la enseñanza del enlace químico está dominada por el uso de modelos para explicar los distintos enlaces, modelos simplificados en exceso, sobre todo en los cursos inferiores, que van subiendo a medida que los estudiantes avanzan de niveles. Por ejemplo, los libros con código 3, 4 y 5, correspondientes a 40. de ESO explican la formación del enlace metálico por atracción de los electrones de valencia y los cationes, y en los cursos superiores (código 10 y 11) se explica con la teoría del mar de electrones y la teoría de bandas. Lo mismo ocurre con la Teoría de Electrones de Valencia (TEV), Teoría de Repulsión Pares de Electrones de la Capa de Valencia (TRPECV) y de hibridación, que son utilizadas sólo en el curso superior (20. de BAC) para explicar el enlace covalente. Cabe destacar que ninguno de los libros, ni los del curso superior, utiliza la Teoría de Orbitales Moleculares (том). Este hecho puede explicarse por el distinto nivel de los alumnos en los diferentes cursos, pero de acuerdo con Levy et al. (2008) muchos de los errores conceptuales relacionados con el enlace químico se deben a la simplificación y generalización de los modelos utilizados en los libros de texto, que presentan una visión limitada e incorrecta del enlace y dan lugar a "impedimentos de aprendizaje". Como se observa en la tabla IV, prácticamente todos los libros de texto analizados utilizan el diagrama de Lewis para la representación del enlace covalente, tanto en los niveles inferiores como en los superiores, pero sólo uno de los libros (código 10) lo emplea también para el enlace iónico.

Con frecuencia los estudiantes confunden los modelos con la realidad (Grosslight, Unger, Jay y Smith, 1991) posiblemente debido a que en la mayoría de los casos los modelos se presentan como si fueran el fenómeno en sí mismo. Por ejemplo, se explica que los metales están constituidos por electrones libres y por cationes, pero no se dice que esto es un modelo teórico y no una realidad. Ninguno de los libros de texto analizados resalta el hecho de que se pueden utilizar distintos modelos para explicar el enlace químico y que los modelos se han ido modificando y cambiando a lo largo de la historia. Así, por ejemplo, el modelo del mar de electrones explica la conductividad eléctrica de los metales por la movilidad de los electrones, pero ésta también puede ser explicada mediante la Teoría de bandas. La coexistencia de modelos confunde a los estudiantes, pues no distinguen entre modelo y realidad (Treagust, 
Chittleborough y Mamiala, 2002), lo que puede deberse a que en los libros no se explica en ningún momento la naturaleza de los modelos, ni sus funciones y limitaciones.

\subsection{Estudio de las fuerzas intermoleculares}

Tabla VIII. Análisis de los libros de textos en relación tratamiento dado al estudio de las fuerzas intermoleculares

\begin{tabular}{|c|c|c|c|c|c|c|c|c|c|c|c|c|}
\hline \multirow{2}{*}{\multicolumn{2}{|c|}{ ítems }} & \multicolumn{11}{|c|}{ Códigos } \\
\hline & & \multirow{2}{*}{\begin{tabular}{|c|}
1 \\
$x$
\end{tabular}} & \multirow{2}{*}{$\begin{array}{l}\frac{2}{x} \\
\end{array}$} & \multirow{2}{*}{$\frac{3}{x}$} & $\frac{4}{y}$ & \multirow[t]{2}{*}{5} & \multirow[t]{2}{*}{6} & \multirow[t]{2}{*}{7} & \multirow[t]{2}{*}{8} & \multirow[t]{2}{*}{9} & \multirow[t]{2}{*}{\begin{tabular}{|l|l}
10 \\
\end{tabular}} & \multirow[t]{2}{*}{11} \\
\hline 1 & No define las fuerzas intermoleculares & & & & $x$ & & & & & & & \\
\hline 2 & $\begin{array}{l}\text { Define fuerzas intermoleculares como fuerzas-unión } \\
\text { entre moléculas }\end{array}$ & & & & & $x$ & & $x$ & & & $x$ & $x$ \\
\hline 3 & $\begin{array}{l}\text { Define las fuerzas intermoleculares como enlace en los } \\
\text { que participan moléculas }\end{array}$ & & & & & & $\mathrm{x}$ & & $x$ & $x$ & & \\
\hline 4 & $\begin{array}{l}\text { No considera a las fuerzas intermoleculares como un tipo } \\
\text { de enlace }\end{array}$ & & & & & $x$ & & $x$ & & & $x$ & \\
\hline 5 & $\begin{array}{l}\text { Clasifica las fuerzas intermoleculares en enlace dipolo- } \\
\text { dipolo, enlace de hidrógeno y enlace dipolo instantáneo- } \\
\text { dipolo inducido }\end{array}$ & & & & & & $\mathrm{x}$ & & $x$ & & $x$ & \\
\hline 6 & $\begin{array}{l}\text { Clasifica las uniones intermoleculares en uniones de Van } \\
\text { der Waals y uniones o enlace de hidrógeno }\end{array}$ & & & & & & & $x$ & & $x$ & & $x$ \\
\hline 7 & $\begin{array}{l}\text { Introduce el término Fuerzas de London para uniones } \\
\text { entre moléculas apolares }\end{array}$ & & & & & & & & & $x$ & $x$ & \\
\hline 8 & $\begin{array}{l}\text { Introduce las propiedades de las sustancias moleculares } \\
\text { referidas a estados de agregación y fuerzas que } \\
\text { mantienen unidas las moléculas }\end{array}$ & $x$ & & & & & & & & & & \\
\hline
\end{tabular}

Los resultados de la tabla VIII muestran cómo los libros de los cursos inferiores no definen las fuerzas intermoleculares (códigos 1-4). El resto de los libros sí las define, y de ellos los de códigos 8, 9 y 11 las consideran un tipo de enlace. Sólo tres de los libros analizados (códigos 6, 8 y 9) definen las fuerzas intermoleculares como enlaces en los que participan las moléculas. Resalta el hecho de que el libro de código 6 define las fuerzas intermoleculares como enlaces en los que participan las moléculas, pero al clasificar los enlaces no incluye las fuerzas intermoleculares como un tipo de enlace y, al contrario, el libro de código 11 las clasifica como un tipo de enlace, pero las define como "fuerzas de atracción entre moléculas". Levy et al. (2008) proponen un nuevo marco para la enseñanza del enlace químico en el que los distintos tipos de enlace se presenten dentro de una escala continua en la que aumenta la fuerza del enlace desde las fuerzas de Van der Waals hasta el enlace iónico. La formación de las interacciones dipolares al igual que el enlace iónico, se explica por la existencia de fuerzas electrostáticas aunque más débiles. El enlace de hidrógeno puede considerarse como una situación intermedia entre las interacciones dipolares y el enlace covalente, y requiere para su explicación un modelo cuántico ya que hay una compartición electrónica considerable.

De nuevo destaca que la terminología utilizada es muy diferente, desde el término más tradicional de "fuerzas intermoleculares" pasando por "fuerzas de Van der Waals" hasta "enlaces intermoleculares". Como ya se indicó antes, una misma editorial utiliza una definición distinta en cada curso. Así, en el libro con código 1 se definen como "Fuerza de unión entre las moléculas", mientras que en el de código 3 lo hace como "enlaces entre moléculas", aun siendo de la misma editorial. Las clasificaciones que se hacen de los distintos tipos de fuerzas intermoleculares son muy diferentes y difieren incluso dentro de una misma editorial en los distintos cursos, clasificación que se complica en el último curso de bachillerato, en el que se estudian con mayor profundidad este tipo de uniones (códigos 9 al 11). 


\section{Conclusiones}

El hecho de que el enlace químico se trate en los diferentes niveles educativos implica para los autores de los libros de texto tener que tomar decisiones sobre qué enseñar, cuándo enseñarlo y qué terminología utilizar en cada nivel. En este estudio observamos que las soluciones adoptadas por cada editorial son muy diferentes. Se ha elaborado una rúbrica que se ha empleado para analizar los contenidos según seis categorías, y que permitirá a los docentes evaluar el planteamiento del tema enlace químico en cualquier libro de texto. Del análisis de los principales libros de texto utilizados en España en la Eso y BAc con base en esa rúbrica, se concluye que casi todas las editoriales incurren, en mayor o menor medida, en confusiones terminológicas, conceptuales y epistemológicas similares. Aunque no existe gran diferencia en la secuenciación de los contenidos, sí se observa confusión en la terminología utilizada, no sólo de una editorial a otra sino incluso dentro de una misma editorial. Con frecuencia se presentan términos que no han sido definidos o explicados, y se usan diferentes terminologías para referirse al mismo concepto. Las mayores diferencias se observan en la terminología empleada en la clasificación de las sustancias según el tipo de enlace y en el estudio de los distintos tipos de fuerzas intermoleculares.

Por todo esto, aconsejamos a los docentes emplear la rúbrica elaborada en este trabajo para analizar el libro de texto a utilizar en sus clases, dada la importancia de la elección acertada del mismo. Por otro lado, sería recomendable que las editoriales unifiquen criterios en la elaboración de los libros de texto, principalmente en cuanto a la terminología utilizada en los distintos niveles educativos.

La forma de enseñar el enlace químico y el tratamiento que del mismo se hace en los libros de texto debería cambiar y estar más de acuerdo con los resultados de la investigaciones realizadas sobre las ideas alternativas de los estudiantes y las dificultades de aprendizaje del enlace químico. Ante los resultados obtenidos en el presente trabajo, el reto es diseñar una nueva metodología de enseñanza del enlace químico que permita superar las dificultades encontradas.

\section{Referencias}

Abraham, M., Grzybowski, E., Renner, J. y Marek, E. (1992). Understanding and misunderstanding of eight graders of five chemistry concepts found in textbook. Journal of Research in Science Teaching, 29(2), 105120.

Ashkenazi, G. y Kosloff, R. (2006). The uncertainty principle and covalent bonding. Chemical Educator, 11, 66-76.

Bergqvist, A., Drechsler, M., De Jong, O. y Rundgren, S. (2013). Representations of chemical bonding models in school textbooks-help or hindrance for understanding? Chemistry Education Research and Practice, 14, 589-606.

Calvo, M. y Martín, M. (2005). Análisis de la adaptación de los libros de texto de Eso al currículo oficial, en el campo de la Química. Enseñanza de las Ciencias, 23(1), 17-32.

De Posada, J. M. (1999). The presentation of metallic bonding in high school science textbook during three decades: science educational reforms and substantive changes of tendencies. Science Educations, 83(4), 423-447.

Fernández, E., Jiménez, E. y Solano, I. (1997). Análisis del concepto de posición en la enseñanza obligatoria básica. En R. Jiménez y A. Wamba, Avances en la didáctica de las ciencias experimentales (pp. 135-143). Universidad de Huelva.

Furió, C. y Guisasola, J. (1997). Diferencias epistemológicas en la enseñanza habitual de los conceptos de campo y potencial eléctrico. Enseñanza de las Ciencias, 15(2), 259-271. 
Gagliardi, R. y Giordan, A. (1986). La historia de las ciencias: una herramienta para la enseñanza. Enseñanza de las Ciencias, 4(3), 253-258.

Grosslight, L., Unger, C., Jay, E. y Smith, C. (1991). Understanding models and their use in science: conceptions of middle and high school students and experts. Journal of Research in Science Teaching, 28(9), 799-822.

Hurst, M. (2002). How we teach molecular structure to freshmen. Journal of Chemical Education, 79(6), 763-764.

Justi, R. y Gilbert, J. K. (2003). Models and modelling in chemical education. En J. K. Gilbert, R. D. Jong, R. Justi, D. F. Treagust y J. H. Van Driel (Eds.), Chemical education:Towards research based practice (pp. 47-68). Holanda: Kluwer Science \& Technology Education Library.

Levy Nahum, T., Mamlok-Noaman, R., Hofstein, A. y Kronik, L. (2008). A new "bottom-up" framework for teaching chemical bonding. Journal of Chemical Education, 85(12), 1680-1685.

Levy, T., Mamlok-Noaman, R., Hofstein, A. y Taber, K. (2010). Teaching and learning the concept of chemical bonding. Studies in Science Education, 46(2), 197-207.

Matus, L., Benarroch, A. y Nappa, N. (2011). La modelización del enlace químico en libros de texto de distintos niveles educativos. Revista Electrónica de Enseñanza de las Ciencias, 10(1), 178-201.

Matus, L., Benarroch, A. y Perales, F. (2008). Las imágenes sobre enlace químico usadas en los libros de texto de educación secundaria. Enseñanza de las Ciencias, 26(2), 153-172.

Riboldi, L., Pliego, O. y Odetti, H. (2004). El enlace químico: una conceptualización poco comprendida. Enseñanza de las Ciencias, 22(2), 195-212.

Solbes, J. y Vilches, A. (1991). Análisis de la introducción de la teoría de enlaces y bandas. Enseñanza de las Ciencias, 9(1), 53-58.

Taber, K. (2001). Building the structural concepts of chemistry: some considerations from educational research. Chemistry Education:Research and Practice in Europe, 2(2), 123-158.

Treagust, D. F., Chittleborough, G. y Mamiala, T. L. (2002). Students' understanding of the role of scientific models in learning science. International Journal of Science Education, 24(4), 537-368. 